\title{
TRONCO CORONARIO ÚNICO QUE NACE DEL SENO CORONARIO DERECHO
}

\section{SINGLE LEFT MAIN CORONARY ARTERY THAT IS BORN FROM THE RIGHT CORONARY SINUS}

\author{
CAROLINA CHACON ${ }^{1}$, MAURICIO PRIOTTI ${ }^{2}$, MARIANA DOMANICO ${ }^{2}$, FRANCISCO ARA ${ }^{3}$, NICOLÁS FERRERI ${ }^{3}, \mathrm{CINTIA} \mathrm{FOCO}^{3}$, \\ ESTEFANÍA PERETTI ${ }^{3}$, MANUEL TRAGHETTI ${ }^{3}$
}

\section{RESUMEN}

La anomalía más frecuente es el nacimiento de la arteria circunfleja desde el seno coronario derecho, pero algunas publicaciones plantean que más común sería la arteria coronaria derecha naciendo desde el seno coronario izquierdo. gualmente, se ha planteado que la presencia de una arteria coronaria con un nacimiento anómalo se asocia a mayor grado de aterosclerosis. Actualmente la tomografía computada multicorte con 64 canales ofrece un excelente método para detección de las anomalías de las arterias coronarias, pudiendo detectar de manera rápida y mínimamente invasiva.

Palabras clave: circulación coronaria, isquemia miocárdica, muerte súbita.

\section{ABSTRACT}

The most frequent anomaly is the birth of the circumflex artery from the right coronary sinus; however, some publications suggest that the right coronary ar tery would be more common, being born from the left coronary sinus. It has also been suggested that the presence of a coronary artery with an anomalous birth is associated with a greater degree of atherosclerosis. Currently, 64-channel multislice computed tomography offers an excellent method for detecting anomalies of the coronary arteries, and can detect them quickly and minimally invasively.

Keywords: coronary circulation, myocardial ischemia, sudden death.

REVISTA CONAREC 2018;33(147):317-318 | DOI:10.32407/RCON/2018147/0317-0318

\section{INTRODUCCIÓN}

La presencia de anomalías congénitas en el nacimiento de las arterias coronarias es un hecho reconocido desde largo tiempo. Las anomalías de las arterias coronarias son raras, se observan con una frecuencia de 0,5 a 1,5\% en pacientes sometidos a angiografía coronaria'. Al inicio se le daba poca importancia, pero con el avance del conocimiento se han reconocido como causa de síntomas isquémicos y de muerte súbita, especialmente en jóvenes, y posible causa de isquemia en la población adulta ${ }^{2,3}$. La anomalía más frecuente es el nacimiento de la arteria circunfleja desde el seno coronario derecho, aunque algunas publicaciones plantean que más común sería la arteria coronaria derecha naciendo desde el seno coronario izquierdo 4,5 . Igualmente, se ha planteado que la presencia de una arteria coronaria con un nacimiento anómalo se asocia a mayor grado de aterosclerosis $s^{6,7}$.

Cada grupo médico, de acuerdo con las técnicas disponibles y su experiencia, debe utilizar la técnica, preferentemente menos invasiva, de la que disponga para su diagnóstico. La mayor parte de las anomalías coronarias se descubren incidentalmente en una cinecoronariografía (CCG) realizada a pacientes con cardiopatía isquémica. Se conoce que este es el examen más utilizado para el diagnóstico de origen anómalo de arteria coronaria, pero

1 Jefe de Residentes de Cardiología Clínica.

2. Coordinador de Residentes de Cardiología Clínica.

3. Residente de Cardiología Clínica. Sanatorio Delta. Ciudad de Rosario, Santa Fe, Rep. Argentina.

$\triangle$ Correspondencia: Dra. Cintia Foco. Mendoza 1540, S2000BIF Rosario, Santa Fe, Rep.Argentina.cintiafoco@gmail.com.

Los autores declaran no poseer conflictos de intereses.

Recibido: 01/11/2018|Aceptado: 20/12/2018 tiene una limitada utilización para ese fin debido a su característica invasiva. Actualmente la tomografía computarizada multicorte (TCMC) con 64 canales ofrece un excelente método para detección de las anomalías de las arterias coronarias, que las evidencia de manera rápida y mínimamente invasi$v^{8}{ }^{8}$. La importancia del estudio de las arterias coronarias, teniendo en cuenta el cuadro clínico del paciente, y el análisis de la baja incidencia en las anomalías coronarias hacen un tema de gran interés para el cardiólogo.

\section{CASO CLÍNICO}

Paciente masculino de 62 años con hipertensión arterial, diabetes mellitus, extabaquismo, obesidad y sedentarismo. Presentaba una clase funcional previa grado II-III, según la clasificación New York Heart Association, con disnea de 1 año de evolución y antecedentes de angioplastia coronaria con implante de un stent liberador de drogas en tercio distal de coronaria derecha en contexto de un infarto agudo de miocardio evolucionado. Posteriormente tuvo una internación por disnea progresiva hasta clase funcional IV. Al examen físico se presentó con signos de insuficiencia cardíaca global, con rales crepitantes bilaterales y edemas en miembros inferiores. El ecocardiograma de ingreso evidenció una fracción de eyección del ventrículo izquierdo del 30\% producida por severa hipoquinesia global. Se indicó la realización de CCG por refractariedad al tratamiento médico la cual informó: tronco coronario único que nace del seno coronario derecho sin obstrucciones y arteria coronaria derecha con obstrucción severa en segmento medio (Figura 1). Se realizó angioplastia coronaria con dos stent farmacológicos en segmento medio de dicha arteria (Figura 2). Posterior a ello el paciente evoluciona favorablemente.

\section{DISCUSIÓN}

Las anomalías en el origen de las arterias coronarias representan entidades poco consideradas, cuya principal importancia subyace en el riesgo 


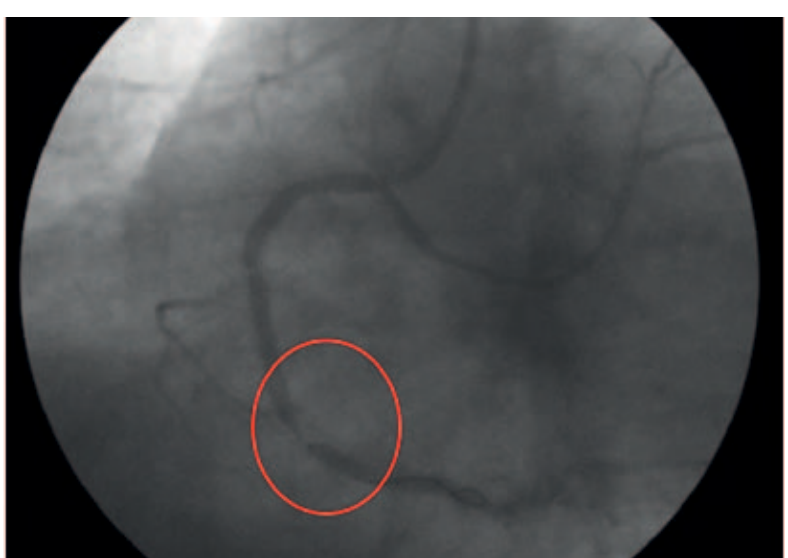

Figura 1. Cinecoronariografía. El círculo rojo señala la arteria coronaria derecha con obstrucción severa en segmento medio.

de desarrollo de isquemia y eventual muerte súbita. Es frecuente que se diagnostiquen incidentalmente en la práctica de una CCG en pacientes afectados por cardiopatía isquémica. Suelen utilizarse otros métodos de imagen para confirmar y/o diagnosticar el trayecto de las arterias coronarias, como la TCMC 64 canales. Siempre se debería intentar definir cuál es el trayecto seguido por la anomalía coronaria y así entregar a los pacientes y a sus médicos recomendaciones, e incluso plantear tratamientos más definitivos ${ }^{9}$. El origen y el trayecto proximal de las arterias coronarias anómalas son los principales factores predictivos de gravedad ${ }^{10}$. Cuando se desarrolla enfermedad arterial coronaria, las consecuencias son significativas y el pronóstico es grave si la obstrucción es proximal, visto que el ostium es único y hay mínima posibilidad de circulación colateral. Muchas teorías se han plasmados con respecto a su asociación con enfermedad coronaria, sea por una marcada angulación presentada por la arteria anómala tras su salida de la aorta", el pasaje interarterial entre la aorta y la arteria pulmonar ${ }^{12}$, los trayectos intramurales o los espasmos coronarios por daño endotelial; todas provocan distintas forma de obstrucción coronaria ${ }^{13}$ que pueden producir isquemia aguda o crónica con la posterior aparición de fibrosis miocárdica, determinando deterioro de la fun-

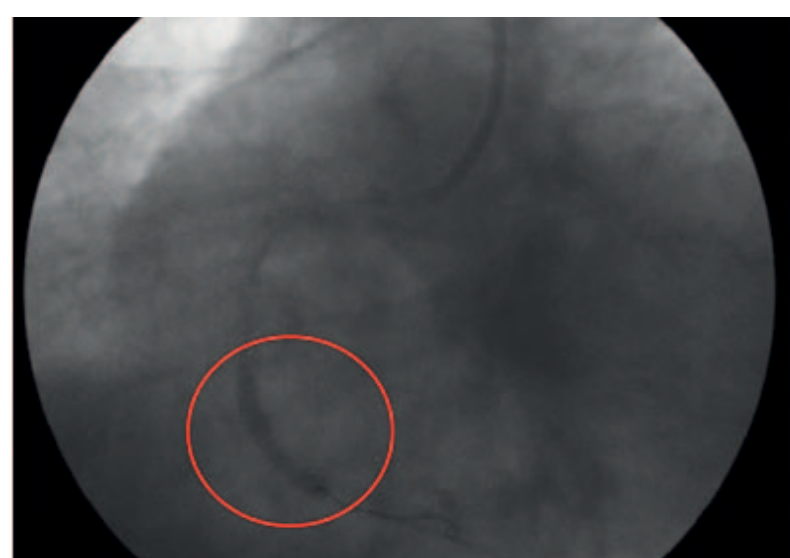

Figura 2. Angioplastia coronaria. El círculo rojo señala la colocación de uno de los stents far macológicos en segmento medio de la arteria coronaria derecha.

ción ventricular con la consiguiente aparición de insuficiencia cardíaca y un aumento marcado en la incidencia de arritmias fatales ${ }^{14}$.

En relación con el caso clínico, se ha decidido la realización de una TCMC para mayor precisión sobre el origen, el curso y la terminación de esos vasos, teniendo en cuenta que la CCG tiene limitación en su proyección. Dado que la anomalía de las arterias coronarias es una entidad relativamente rara, es necesario plantear el adecuado método diagnóstico y el planeamiento terapéutico.

\section{CONCLUSIÓN}

La anomalía en el nacimiento de las arterias coronarias es una entidad de baja prevalencia en nuestro medio, sin embargo su fuerte asociación con cardiopatía isquémica y muerte súbita en adultos jóvenes nos invita a reflexionar si debemos incorporar nuevos métodos diagnósticos para su detección en pacientes asintomáticos así como implementar un registro de casos clínicos a fin de orientar conductas diagnósticas y terapéuticas dirigidas a disminuir la incidencia de arritmias malignas en esta población que dejó de ser aislada.

\section{BIBLIOGRAFÍA}

1. Angelini P, Velasco JA, Flamm S. Coronary anomalies: incidence, pathophysiology and clinical relevance. Circulation 2002; 105(20):2449-54.

2. Kragel $A$, Roberts $W$. Anomalous origin of either the right or left main coronary artery from the aorta with subsequent coursing between aorta and pulmonary trunk: Analysis of 32 necropsy cases. Am J Cardiol 1988;62(10):771-7.

3. Maron BJ. Sudden death in young athletes. N Engl J Med 2003;349(11):1064-75.

4. Garg N, Tewari S, Kapoor A, Gupta D, Sinha N. Primary congenital anomalies of the coronary arteries: a coronary anteriographic study. Int J Cardiol 2000;74(1):39-46.

5. Angelini P, Velasco J, Flamm S. Coronary anomalies. Incidence, pathophysiology and clinical relevance. Circulation 2001;105(20):2449-54.

6. Silverman K, Bulkley B, Hutchins G. Anomalous left circumflex coronary artery: "normal" variant of uncertain clinical and pathologic significance. Am J Cardiol 1978;41(7):1311-4.

7. Click R, Holmes D, Vlietstra R, Kosiski A, Kronmal R. Anomalous coronary arteries: Location, degree of atherosclerosis and effect on survival. A report t from Coronary Artery Surgery Study (CASS). J Am Coll Cardiol 1989;13(3):531-7.

8. Rabelo DR, Barros MVL, Nunes MCP, Oliveira CCC, Siqueira MHA. Angiotomografía coronaria multislice en la evaluación del origen anómalo de las arterias coronarias. Arq Bras Cardiol 2012;98(3)266-72.

9. Doorey A, Pasquale M, Lally I, Mintz G, Marshall E, Ramos D. Six-month success of intracoronary stenting for anomalous coronary arteries associated with myocardial ischemia. Am J Cardiol 2000;86(5):580-2.

10. Dawn B, Talley JD, Prince CR, Hoque A, Morris GT, Xenopoulos NP, et al. Two-dimensional and doppler transesophageal echocardiographic delineation and flow characterization of anomalous coronary arteries in adults. J Am Soc Echocardiogr 2003;16(12):1274-86

11. Virmani R, Chun PK.C, Goldstein RE, Robinowitz M, McAllister HA. Acute takeoffs of the coronary arteries along the aortic wall and congenital coronary ostial valvelike ridges: association with sudden death. J Am Coll Cardiol 1986;3(3):766-71.

12. Bloomfield P, Erhlich C, Folland AD, Bianco J, Tow DE, Parisi AF. Anomalous right coronary artery: a surgically correctable cause of angina pectoris. Am J Cardiol 1983;51(7):1235-7.

13. Frommelt PC, Frommelt MA, Tweddell JS, Jaquiss RD. Prospective echocardiographic diagnosis and surgical repair of anomalous origin of a coronary artery from the opposite sinus with an interarterial course. I Am Coll Cardiol 2003;42(1):148-54.

14. Basso C, Corrado D, Thiene G. Congenital coronary anomalies as an important cause of sudden death in the young. Cardiol Rev 2001;9(6):312-7. 